\title{
A micro-Raman and infrared study of several Hayabusa category 3 (organic) particles
}

\author{
Fumio Kitajima ${ }^{1 *}$, Masayuki Uesugi ${ }^{2}$, Yuzuru Karouji ${ }^{2}$, Yukihiro Ishibashi ${ }^{2}$, Toru Yada ${ }^{2}$, Hiroshi Naraoka', \\ Masanao Abe ${ }^{2}$, Akio Fujimura ${ }^{2}$, Motoo Ito ${ }^{3}$, Hikaru Yabuta ${ }^{4}$, Hajime Mita ${ }^{5}$, Yoshinori Takano ${ }^{6}$ and Tatsuaki Okada ${ }^{2}$
}

\begin{abstract}
Three category 3 (organic) particles (RB-QD04-0001, RB-QD04-0047-02, and RA-QD02-0120) and so-called 'white object' found in the sample container have been examined by micro-Raman and infrared (IR) spectroscopy. In addition, several artificial substances that could occur as possible contaminants and chondritic insoluble organic matter (IOM) prepared from the Murchison CM2 chondrite were analyzed. The Raman spectra of the particles show broad G-band and weak D-band. The G-band parameters plot in the disordered region and close to the artifact produced from a Viton glove after laser exposure rather than chondritic IOM. The particles were therefore originally at low maturity level, suggesting that they have not experienced strong heating and are therefore not related to the LL4-6 parent body. The IR spectra are not similar to that of chondritic IOM. Furthermore, the particles cannot be identified as some artificial carbonaceous substances, including the white object, which are the possible contaminants, examined in this investigation. Although it cannot be determined exactly whether the three category 3 particles are extraterrestrial, the limited IR and Raman results in this investigation strongly suggest their terrestrial origin. Although they could not be directly related to the artificial contaminants examined in this investigation, they may yet be reaction products from similar substances that flew on the mission. In particular, RB-QD04-0047-02 shows several infrared spectral absorption bands in common with the 'white object.' This may relate to the degradation of a polyimide/polyamide resin.
\end{abstract}

Keywords: The Hayabusa spacecraft; Carbonaceous material; Category 3 particle; Micro-Raman spectroscopy; Micro-infrared spectroscopy; Insoluble organic matter

\section{Correspondence/Findings Introduction}

The Hayabusa space craft returned to the Earth on 13 June 2010 from the Muses- $C$ region on the asteroid Itokawa with more than 1,500 tiny particles (e.g., Nakamura et al. 2011). Subsequently, more than 450 particles have been picked up from the sample catcher (Uesugi et al. 2014). The recovered particles have been classified into four categories (Yada et al. 2014). Categories 1 and 2 are siliceous particles, and category 3 particles are carbonaceous. Category 4 particles are artificial materials such as aluminum flakes (Yada et al. 2014), and the so-called 'white object' found in the sample container (http://hayabusaao.isas.jaxa.jp/catalog/contaminations.pdf) may be also included in this category.

\footnotetext{
* Correspondence: kitajima@geo.kyushu-u.ac.jp

${ }^{1}$ Department of Earth and Planetary Sciences, Faculty of Science, Kyushu University, Hakozaki, Fukuoka 812-8581, Japan

Full list of author information is available at the end of the article
}

Category 1 particles are composed only of transparent minerals, whereas category 2 particles consist of not only transparent particles but also opaque minerals (Yada et al. 2014). Particles belonging to categories 1 and 2 have been confirmed as Itokawa regolith (e.g., Ebihara et al. 2011; Nagao et al. 2011; Nakamura et al. 2011; Noguchi et al. 2011; Tsuchiyama et al. 2011; Yurimoto et al. 2011).

The asteroid Itokawa belongs to S-type and its surface has been considered to have an olivine-rich mineral assemblage similar to that of LL5 chondrite (Abe et al. 2006; Hiroi et al. 2006). The returned stony samples confirm that Itokawa is indeed an ordinary chondrite parent body of grade LL4-6 (Nakamura et al. 2011). Although average concentrations of $0.04 \%$ to $0.19 \%$ carbon in matrices of LL4-6 chondrites have been reported (Makjanic et al. 1993), carbonaceous matter in such chondrites is expected to be depleted and thermally altered. However, Itokawa is a rubble-pile object; therefore, primitive carbon-rich fragments could also be 
present, besides more mature carbonaceous matter. Several stony particles were examined in search of such organic materials; however, any amino acids (Naraoka et al. 2012) or insoluble organic matter (IOM) of extraterrestrial origin have not yet been found (Kitajima et al. 2011) on the surface of these particles.

Besides the silicate particles, several carbon-rich particles classified into category 3 have been found in the sample catcher (e.g., Yada et al. 2014; Uesugi et al. 2014). The particles are mainly composed of carbon and often contain oxygen, nitrogen, and sulfur (Yada et al. 2014). It has not yet been clarified whether these particles are extraterrestrial in origin. In this investigation, as a part of the sequential analysis of category 3 particles, we examined three category 3 particles (RB-QD04-0001, RBQD04-0047-02, and RA-QD02-0120) by micro-Raman and Fourier transform infrared (FTIR) spectroscopies, together with several artificial carbonaceous substances including the 'white object' found in the sample container. The artificial substances are analyzed in order to get a clue as to whether the category 3 particles are extraterrestrial or not, and our data should be coupled with the results of other kinds of analyses (NanoSIMS, time-of-flight secondary ion mass spectrometry (ToFSIMS), and X-ray absorption near edge structure (XANES) described in a series of articles in this issue (Uesugi et al. 2014; Ito et al. 2014; Yabuta et al. 2014; Naraoka et al. 2015).

\section{Samples}

\section{Category 3 particles}

Out of 457 recovered particles, 58 have been classified into category 3 (Uesugi et al. 2014). The particles were mainly composed of $\mathrm{C}, \mathrm{N}$, and $\mathrm{O}$ and further classified into three subtypes, types 1 to 3 , as blocky, fibrous, and faint, respectively (Uesugi et al. 2014). Three category 3 particles (RB-QD04-0001, RB-QD04-0047-02, and RAQD02-0120) were analyzed in this study. RB-QD040001 belongs to faint type. It is $19 \mu \mathrm{m}$ in size. C, $\mathrm{N}$, and $\mathrm{O}$ are observed by FE-SEM-EDS. RB-QD04-004702 belongs to blocky type, $28 \mu \mathrm{m}$ in size, and contains $\mathrm{C}$ and O. RA-QD02-0120 also belongs to the blocky type, $26 \mu \mathrm{m}$ in size, and contains $\mathrm{C}$ and $\mathrm{O}$. Further analyses by TEM/STEM (Uesugi et al. 2014), N-XANES (Yabuta et al. 2014), and $\mathrm{H}, \mathrm{C}$, and $\mathrm{N}$ isotopic investigation (Ito et al. 2014) revealed the presence of $\mathrm{N}$ in the RBQD04-0047-02 and RA-QD02-0120 particles. A detailed description of the category 3 particles is given in Uesugi et al. (2014). Because the particles must be fixed in some manner for the NanoSIMS and ToF-MS analyses planned as parts of a sequential analysis, they were pressed onto gold plates (Uesugi et al. 2014) at the PMSCF/JAXA (The Planetary Material Sample Curation Facility of JAXA). The particles RA-QD02-0120 and RB-QD04-0047-02 were also investigated by XANES analyses (Yabuta et al. 2014). Calcium carbonate inclusions of up to $300 \mathrm{~nm}$ have been identified in RA-QD02-0120 by TEM/STEM (Uesugi et al. 2014) as well as $\mathrm{C}$ and $\mathrm{Ca}$ by XANES (Yabuta et al. 2014). The particle RB-QD04-0047-02 was also analyzed by ToFSIMS (Naraoka et al. 2015); TEM/STEM observation (Uesugi et al. 2014) and ToF-SIMS analysis pointed out a wide but heterogeneous distribution of $\mathrm{Si}$. One particle, RB-QD04-0001, was allocated in the initial analysis, and the intact particle had also been examined using a diamond holder previously. Although the collected spectra have not been definitive, $\mathrm{O}-\mathrm{H}$ stretching at $3,239 \mathrm{~cm}^{-1}$ and several absorption bands in 1,700 to $1,277 \mathrm{~cm}^{-1}$ region have been observed. These absorption bands may be in common with RA-QD02-0008. The results of the analysis using the diamond holder will be reported in detail elsewhere. Because solvent extraction has not been investigated, whether the particles are soluble or insoluble in organic solvents has not yet been clarified.

\section{Artificial carbonaceous substances and the 'white object'}

The clean chambers set up at PMSCF/JAXA are managed to restrict materials only to stainless steel (304 and 316), pure aluminum and aluminum alloy (A6061), quartz glass, PTFE (polytetrafluoroethylene), and Viton (DuPont Performance Elastomers L.L.C., Wilmington, DE, USA) (Yada et al. 2014). The skeletal structures of PTFE (compound I) and Viton (compound II) are shown in Figure 1. Some parts of the sample container of Hayabusa such as the rotational cylinder were treated with a special Tufram coating (General Magnaplate Corporation, Linden, NJ, USA). It was performed in such a way that the surface of the aluminum alloy was treated to be alumite and coated with Teflon to obtain smooth movement (Yada et al. 2014). The structure of the sample container is described in Yada et al. (2014). In this investigation, we examined Viton glove (two samples named CC\#7 and CC\#8 collected from different positions of the clean chambers in PMSCF/JAXA). The white object found in the sample container was compared to Tufram and Kapton 120HR161, a polyimide resin, as possible carbonaceous contaminants. Kapton 120HR161 is a material coated with perfluoroethylenepropene (FEP; its skeletal structure is given in Figure 1, compound III) on the surface of Kapton $\mathrm{H}$ (its skeletal structure is shown in Figure 1, compound IV). The 'white object' has been proposed to be a mixture of polyamide generated through hydrazinolysis of a polyimide resin and a small amount of polyimide which is the starting material (http://hayabusaao.isas. jaxa.jp/catalog/contaminations.pdf). 
$\mathbf{a}$ ) $\left.-\mathrm{CF}_{2}-\mathrm{CF}_{\mathbf{2}^{-}}-\mathrm{CF}_{\mathbf{2}^{-}}\right)$

I

b<smiles>C=[C+]C(C)(C)C</smiles>

II c<smiles>[3H][C+][CH+]C(C)(C)[Te]</smiles>

d<smiles>CCn1c(=O)c2cc3c(=O)n(-c4ccc(Oc5ccc(C)cc5)cc4)c(=O)c3cc2c1=O</smiles>

IV

e<smiles>CCNC(=O)c1cc(C(=O)Nc2ccc(Oc3ccc(C)cc3)cc2)c(C(=O)O)cc1C(=O)O</smiles>

f<smiles>CNC(=O)c1cc(C(=O)Nc2ccc(Oc3ccc(C)cc3)cc2)c(C(=O)NN)cc1C(N)=O</smiles><smiles>CNC(=O)c1cc2c(=O)[nH][nH]c(=O)c2cc1C(=O)NN</smiles>

VII

\section{VIII}

Figure 1 Chemical structure of the compounds referred in the text. Skeletal structures of (a) PTFE (polytetrafluoroethylene) and Viton rubber. (b) $\mathrm{CF}_{3}-$ can be replaced by $\mathrm{CF}_{3} \mathrm{O}$-, and up to several percent of fluorine can be replaced by hydrogen. (c) FEP (perfluoroethylenepropene). (d) Kapton $\mathrm{H}$, a polyimide resin. (e) Poly(amic acid), a starting material for industrial production of the compound IV. (f) Hydrazide, a hydrazinolysis product of the polyimide resin IV. The polyimide resin IV generates $\mathbf{( g )}$ arylether VII and (h) hydrazide VIII via (f) hydrazide VI in the presence of hydrazine.

\section{Chondritic IOM}

At least two types of extraterrestrial organic materials can be expected in the Itokawa particles. One is a thermally mature material that would have been contained in the original Itokawa parent body. The other is a rather primitive carbonaceous material that would be transported from space. Because IOM from the LL4-6 chondrites is scarce (in types 3.6 to 3.7 ordinary chondrites, most IOM has disappeared; Busemann et al. 2007), the IOM sample was prepared from the Murchison CM2 chondrite as a primitive extraterrestrial material for comparison. The preparation method is described in Oba and Naraoka (2009). The sample was pressed onto a copper plate for the measurement at Kyushu University. 


\section{Methods}

One category 3 particle, RA-QD02-0120, was analyzed at PMSCF/JAXA. The other particles were analyzed at Kyushu University. The particle RB-QDO4-0001 had been crushed and scattered on the gold plate; therefore, two regions named RB1 and RB2 were analyzed. Mid-IR reflectance spectra of RA-QD02-0120 were obtained using a JASCO FTIR-6100 spectrometer with a mercurycadmium-telluride (MCT) detector at PMSCF/JAXA. A total of 1,024 scans of spectra were accumulated with a $25 \times 25 \mu^{2}$ aperture. The IR spectra of the other samples were collected using a Perkin-Elmer Spectrum One spectrometer (PerkinElmer Inc., Waltham, MA, USA) with a MCT detector. The same total scans of the spectra were accumulated with the same aperture size. The reflectance spectra of the category 3 particles on gold plate and the IOM from the Murchison chondrite on the copper plate were collected. The reflectance spectra of Tufram were obtained by measuring the surface of a spare rotational cylinder of the Hayabusa sample container. The transmission spectra of the Viton rubber and the white object on diamond plate $(0.2 \mu \mathrm{m}$ thickness) were also collected. Commercial Kapton 120HR616 film was analyzed without the diamond plate. IR measurements were performed prior to the Raman analyses to avoid sample damage that would be caused by laser exposure. Raman spectra were obtained using JEOL JRS system 2000 Raman spectrometer (JEOL Ltd., Tokyo, Japan) (1,800 lines/mm grating). An excitation wavelength of $514.5 \mathrm{~nm}$ was used on an argon ion laser. The laser beam was focused by a microscope equipped with $\mathrm{a} \times 50$ or $\times 100$ objective, leading to a spot diameter of 2 or $1 \mu \mathrm{m}$. Three scans of spectra of the Murchison IOM were accumulated with a 10-s exposure. A scan of spectrum of the Viton rubber with a 10-s exposure was also obtained. The laser power at the outlet of the light source was $20.3 \mathrm{~mW}$, and on the sample surface was $1.0 \mathrm{~mW}$. Five scans of spectra of the two category 3 particles, RB-QD04-0047-02 and RB-QD04-0001, were accumulated with a 2-s exposure. Five points were analyzed for each particle (five points for each region from RB-QD040001). The power on the sample surface was $0.2 \mathrm{~mW}$, which was reduced more than usual because the particles might be sensitive to heating. Following analysis, we observed that no hole was burnt through the sample surface and the spectral features did not transform during accumulation; therefore, we consider that laser damage is negligible (five scans with a 2-s exposure at the power of $0.2 \mathrm{~mW}$ on the sample surface did not show laser-induced alteration of the Viton rubber). Raman spectrum of RAQD02-0120 was obtained with a JASCO NRS-5100 Raman spectrometer (JASCO Corporation, Tokyo, Japan) (1,800 lines/mm grating) at PMSCF/JAXA. An excitation wavelength of $532.18 \mathrm{~nm}$ was used on an argon ion laser. The laser beam was focused by a microscope equipped with $a \times 50$ objective. The power on the sample surface was lower than $0.1 \mathrm{~mW}$. Ten scans of the spectra with $30 \mathrm{~s}$ exposure were accumulated. All measurements were performed in the atmosphere. The curve fitting for the Raman spectra was performed using the pseudo-Voigt function after subtraction of the linear baseline.

\section{Results and discussion \\ The category 3 particles}

The Raman spectrum indicates the structural order of carbonaceous matter. Ideal monocrystalline graphite shows only the so-called G-band at $1,581 \mathrm{~cm}^{-1}$. Depending on the level of disorder, the G-band shifts to higher wavenumbers and can be observed at around $1,600 \mathrm{~cm}^{-1}$ (e.g., Sandford et al. 2006; Busemann et al. 2007; Quirico et al. 2009). The so-called D-band at around $1,350 \mathrm{~cm}^{-1}$ is not present in the perfectly stacked graphite and it is induced by structural defects. Its size reflects increasing disorder (Busemann et al. 2007; Quirico et al. 2009).

Figures 2, 3 and 4 show a few representative Raman spectra of the three category 3 particles. The Raman spectra show broad G-band and weak D-band, although the relative intensity varies depending upon location. In the initial analysis using the diamond holder, the particle RB-QD04-0001 showed neither G- nor D-band but only a high fluorescence background. However in region RB1 analyzed in this work, the bands were present. This may be the result of the use of the short, workingdistance objective lens.

Figure 5 shows several IR spectra of the three category 3 particles. It should be noted that the absence of IR aromatic absorption bands does not always indicate the absence of the aromatic skeleton because IR will not detect highly condensed graphene structure (Kebukawa et al. 2011), which shows Raman G- and D-bands. All the category 3 particles, in particular, RB-QD04-0047-02 and RA-QD02-0120, showed broad O-H stretching in the region 3,503 to 3,300 $\mathrm{cm}^{-1}$. RB-QD04-0047-02 also showed fine structures in the 1,729 to $965 \mathrm{~cm}^{-1}$ region. The absorption at $1,729 \mathrm{~cm}^{-1}$ is assigned to $\mathrm{C}=\mathrm{O}$ stretching, and the absorption at $1,506 \mathrm{~cm}^{-1}$ is assigned to aromatic $\mathrm{C}=\mathrm{C}$. The absorption band at around $1,000 \mathrm{~cm}^{-1}$ may be by the $\mathrm{Si}-\mathrm{O}$ stretching, corresponding to the $\mathrm{Si}$ in the sample (Uesugi et al. 2014; Naraoka et al. 2015). This will be discussed further along with the results from the white object in the 'The category 3 particles as thermally unaltered materials' section. RBQD04-0001_RB1 shows weak absorptions at 1,684 $\mathrm{cm}^{-1}$ ( $\mathrm{C}=\mathrm{O}$ stretching), and $1,509 \mathrm{~cm}^{-1}$ (aromatic $\mathrm{C}=\mathrm{C}$ stretching). These absorption bands had been suggested by the initial analysis using the diamond holder. However, this particle had been crushed and scattered on the gold plate, and so additional information could not be obtained. The IR spectral features of the two particles 


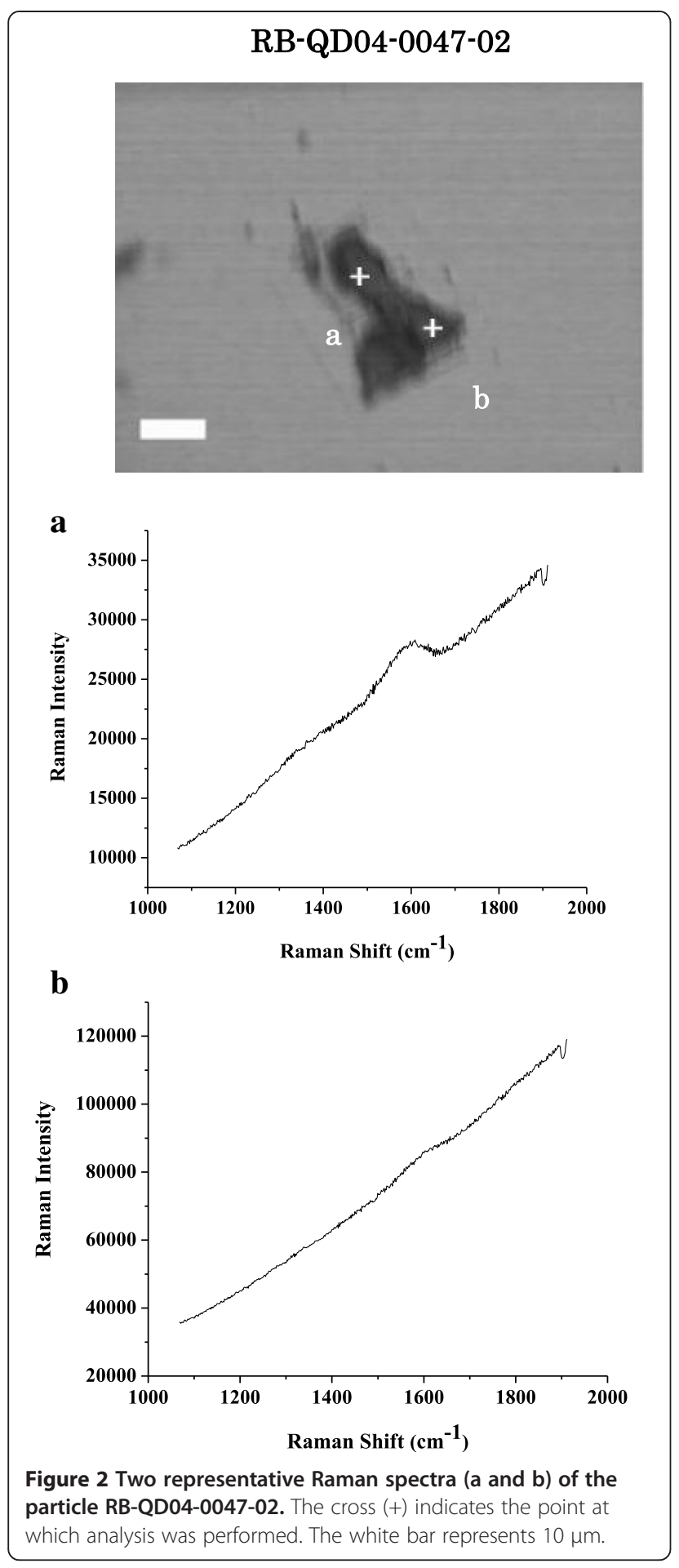

are different from that of chondritic IOM as shown in the 'Chondritic IOM' section. Although these two particles show $\mathrm{C}=\mathrm{O}$ stretching, they lack aliphatic $\mathrm{C}-\mathrm{H}$ stretching usually observed in chondritic IOM. RAQD02-0120, on the contrary, shows aliphatic C-H stretchings around $2,994 \mathrm{~cm}^{-1}$ together with $\mathrm{O}-\mathrm{H}$ stretching at $3,342 \mathrm{~cm}^{-1}$. The absorption band at around
$1,645 \mathrm{~cm}^{-1}$ may be assigned to $\mathrm{C}=\mathrm{O}$ stretching. These absorption bands are, however, weak and unclear, partly because it may be related to the sample alteration caused by NanoSIMS analysis (Uesugi et al. 2014).

\section{Chondritic IOM}

Figure 6 shows the IR and Raman spectra of the chondritic IOM prepared from the Murchison meteorite. The typical IR spectrum of IOM from carbonaceous chondrites is characterized by $\mathrm{O}-\mathrm{H}$ stretching at around $3,400 \mathrm{~cm}^{-1}$ (being due to alcohol, carboxyl, and water), aliphatic $\mathrm{C}-\mathrm{H}$ stretchings in 2,958 to $2,855 \mathrm{~cm}^{-1}$, and carbonyl stretchings in the region at 1,725 to $1,665 \mathrm{~cm}^{-1}$ (e.g., Kebukawa et al. 2011). The observed IR spectrum is characterized by the $\mathrm{O}-\mathrm{H}$ stretching band at a frequency of $3,515 \mathrm{~cm}^{-1}$, the $\mathrm{C}-\mathrm{H}$ stretchings at 2,961 (aliphatic $\mathrm{CH}_{3}$ : $\mathrm{C}-\mathrm{H}$ asymmetric stretching), 2,931 (aliphatic $\mathrm{CH}_{2}: \mathrm{C}-\mathrm{H}$ asymmetric stretching), and $2,872 \mathrm{~cm}^{-1}$ (aliphatic $\mathrm{CH}_{3}$ : $\mathrm{C}-\mathrm{H}$ symmetric stretching), and carbonyl stretching band at $1,716 \mathrm{~cm}^{-1}$ in accordance with some previous reports (e.g., Kebukawa et al. 2010, 2011). The observed raw Raman spectra are consistent with the literature data (Quirico et al. 2009).

\section{White object}

Figure 7a shows the so-called 'white object' found in the sample container. It seems needle-like but also powdery and white to slightly brown colored. Figure $7 \mathrm{~b}$ shows its IR spectrum. It has been proposed that the object is a mixture of polyamide and a small amount of polyimide. The polyamide is a hydrazinolysis product of the polyimide resin, the starting material (http://hayabusaao.isas. jaxa.jp/catalog/contaminations.pdf). While a detailed structure is not given on the website, it must have such structures as depicted in Figure 1. Compound IV in Figure 1 shows the unit structure of Kapton $\mathrm{H}$, a typical polyimide resin, and compound $\mathrm{V}$ is poly(amic acid), a starting material for industrial production of compound IV. Hydrazinolysis of polyimide would give rise to hydrazide (compound VIII in Figure 1) and compound VII via hydrazide compound VI (Ueno et al. 2009). The carbonyl absorption band at $1,722 \mathrm{~cm}^{-1}$ indicates imide $\mathrm{C}=\mathrm{O}$ stretching; the absorption at $1,650 \mathrm{~cm}^{-1}$ indicates amide (I) $\mathrm{C}=\mathrm{O}$ stretching; absorption at $1,543 \mathrm{~cm}^{-1}$ suggests $\mathrm{N}-\mathrm{H}$ bending (secondary amide II). The absorption at $1,219 \mathrm{~cm}^{-1}$ with a shoulder suggests $\mathrm{C}-\mathrm{O}$ and/or C-F (some kinds of Kapton are coated with FEP). The broad absorption around $3,051 \mathrm{~cm}^{-1}$ suggests aromatic $\mathrm{C}-\mathrm{H}$ stretching. Aromatic $\mathrm{C}=\mathrm{C}$ stretchings are observed at 1,498 and $1,606 \mathrm{~cm}^{-1}$. The sharp and extraordinarily strong aromatic $\mathrm{C}=\mathrm{C}$ stretching at $1,498 \mathrm{~cm}^{-1}$ is remarkable for this substance compared with the commercial Kapton (Figure 7c). It may be a contribution of hydrazide VIII and/or arylether VII. The absorption 


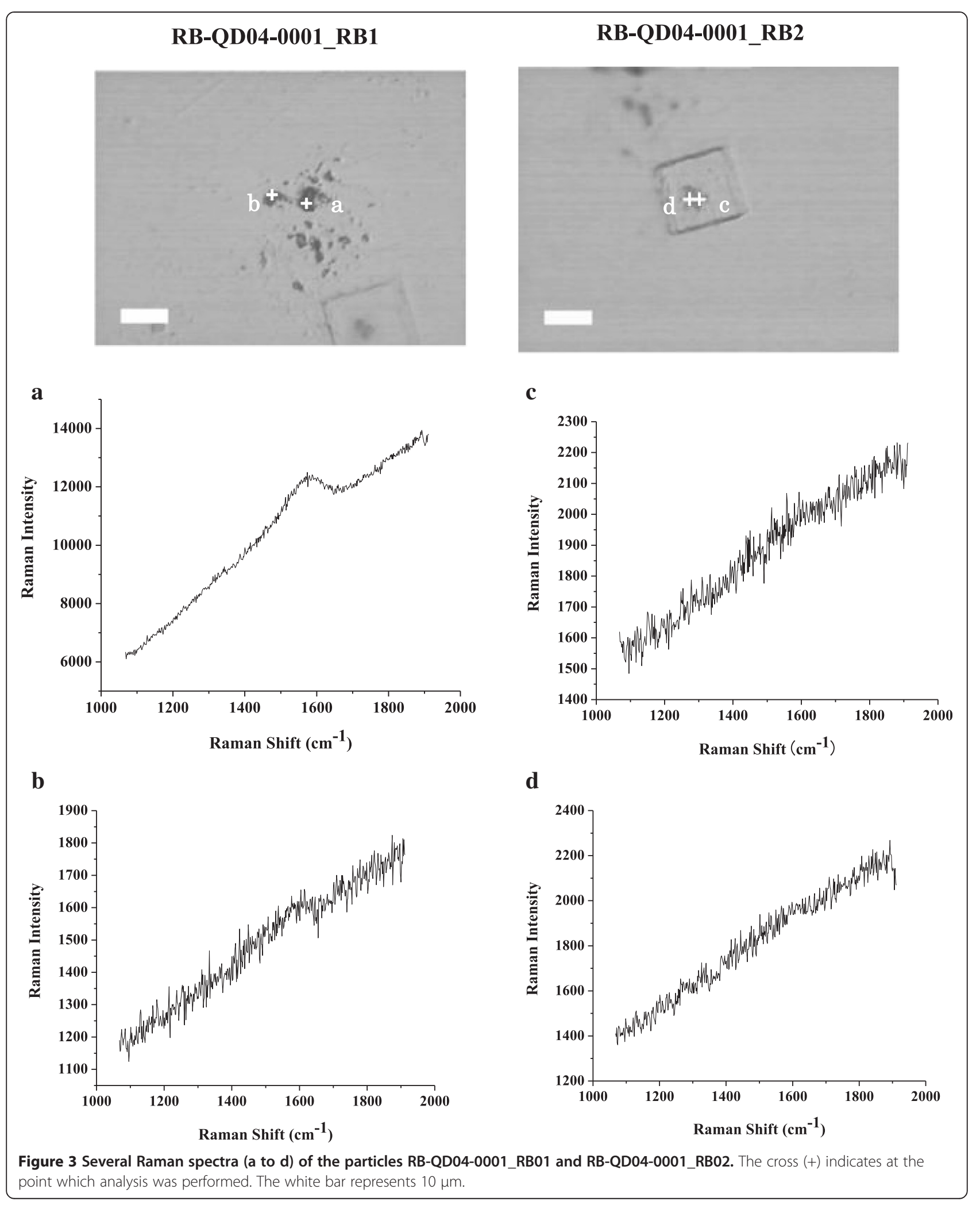




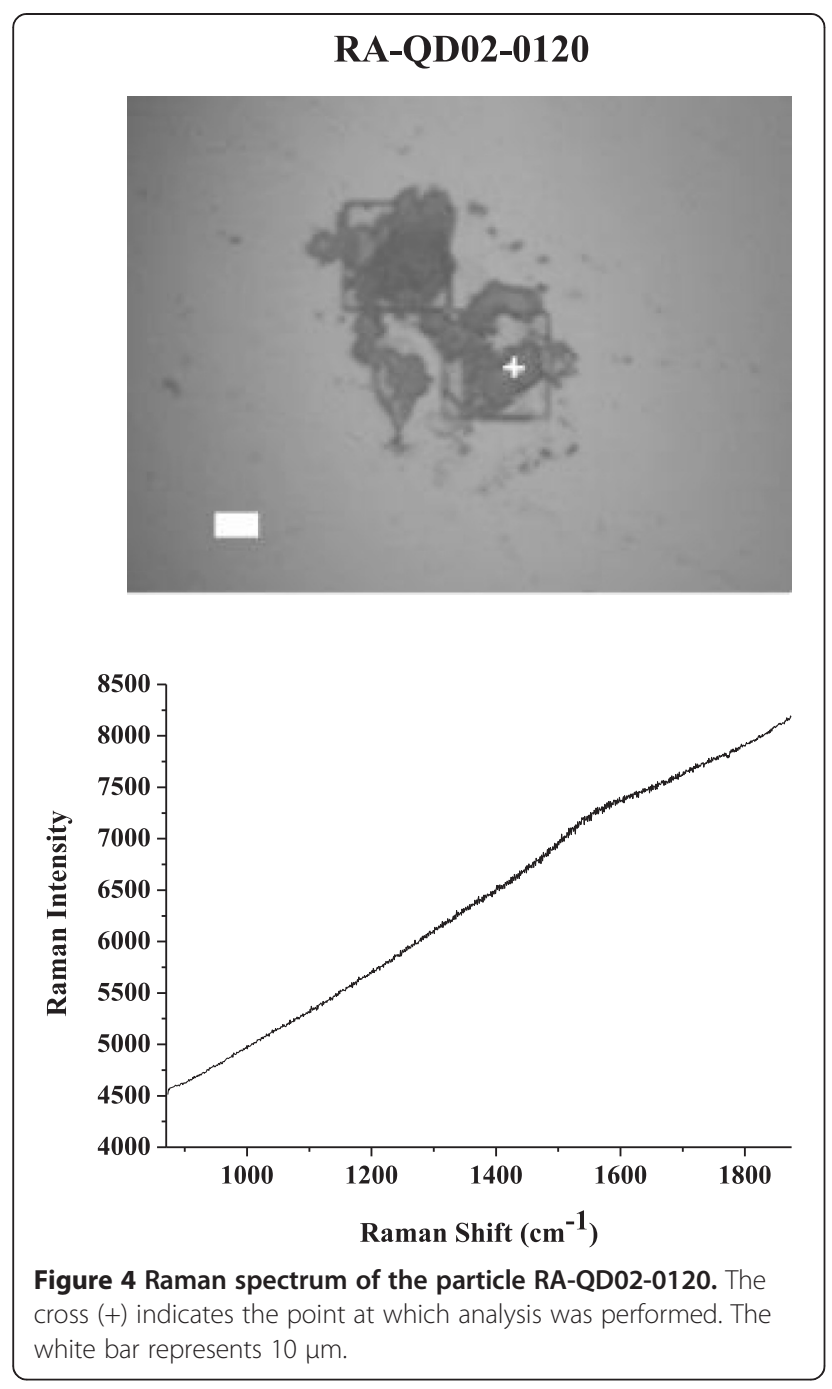

band at $3,243 \mathrm{~cm}^{-1}$ suggests the presence of the $\mathrm{O}-\mathrm{H}$ functional group such as those of compound $\mathrm{V}$ and its presence rather than predominance of hydrazide VI and VIII. Alternatively, the $\mathrm{O}-\mathrm{H}$ absorption may be accounted for by its hygroscopic nature.

\section{Viton glove (CC2\#8)}

Figure $8 \mathrm{a}$ shows the IR spectrum of the Viton glove (named CC2\#8). It is a possible contaminant. The C-F stretching is observed at the frequency of $1,151 \mathrm{~cm}^{-1}$. The absorption at $1,016 \mathrm{~cm}^{-1}$ indicates $\mathrm{Si}-\mathrm{O}$ stretching of talc as additions. This material is very sensitive to heating, and excess exposure to laser results in artificial G- and D-bands (Figure 8b). According to the skeletal structure of Viton (compound II in Figure 1), which does not have the graphene structure, the observed Raman bands are apparently an artifact. Another Viton glove sample (named CC2\#7) showed essentially the same results.

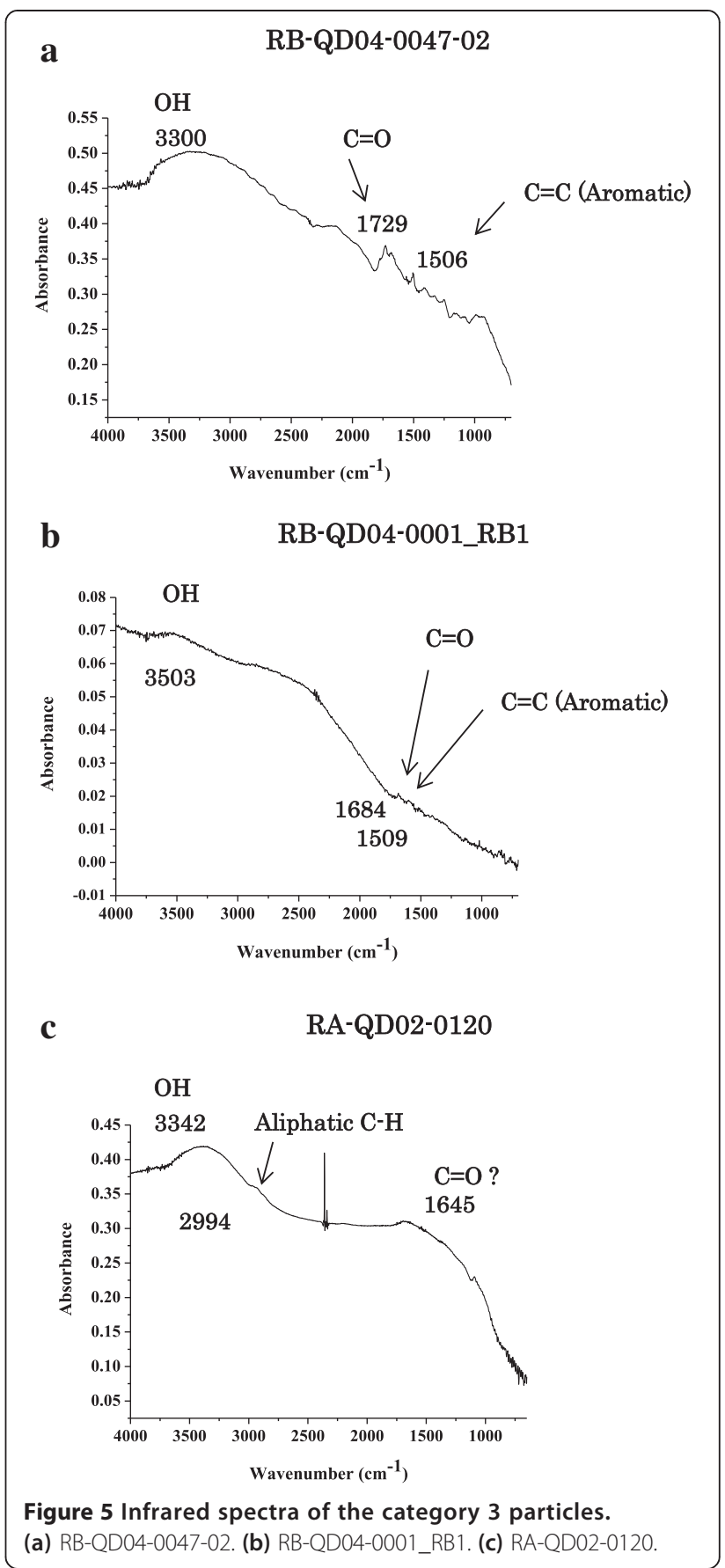

The IR spectra of the category 3 particles differ from that of Viton glove in the absence of C-F stretching at $1,151 \mathrm{~cm}^{-1}$. F-XANES analysis of RB-QD04-0047-02 shows the absence of fluorine (Yabuta et al. 2014), being consistent with this IR observation. Therefore, the particles are, at least, not 'fresh' fragments of the Viton rubber itself.

\section{Tufram}

Figure 8c shows the IR spectrum of Tufram that is used for the surface coating of the rotational cylinder of the 


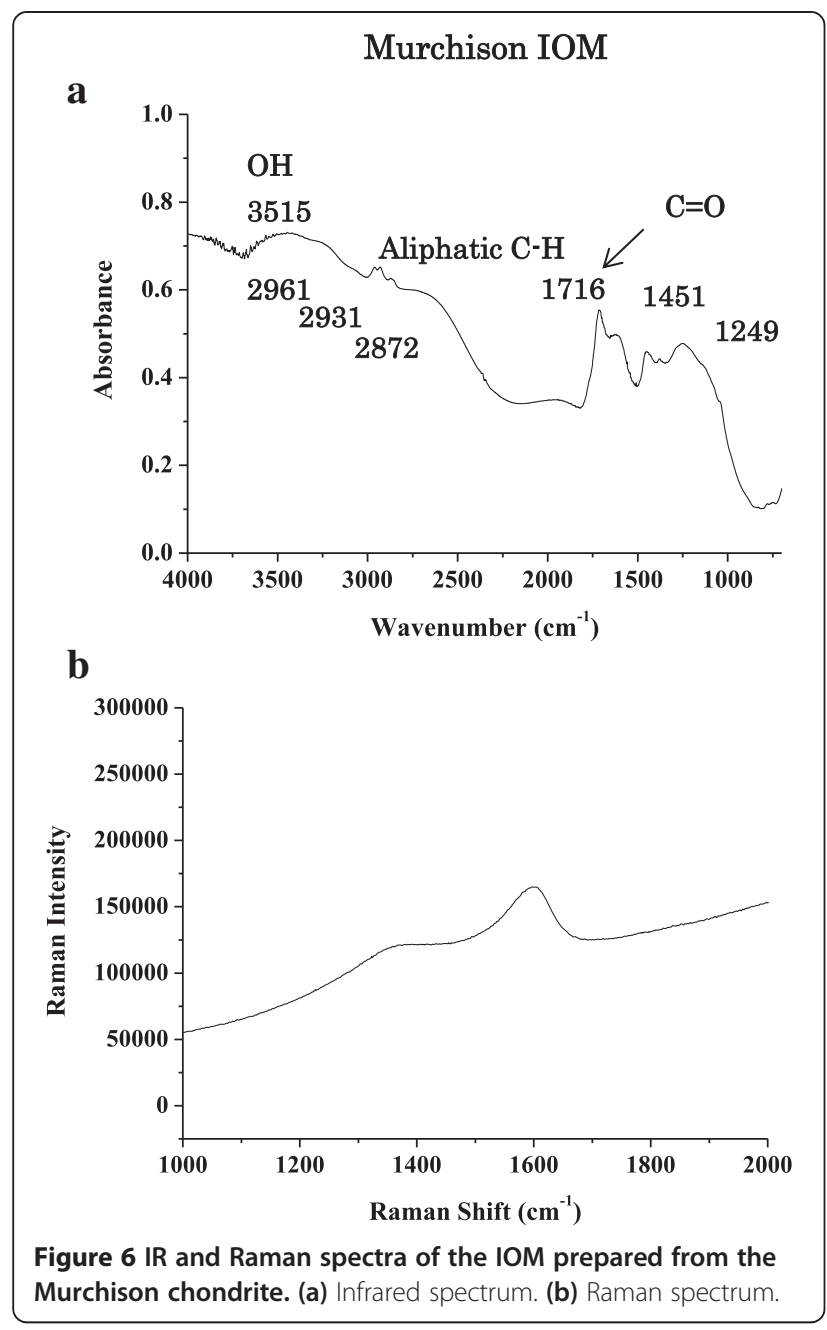

Hayabusa sample container. The strong absorption band at $1,259 \mathrm{~cm}^{-1}$ of C-F stretching of PTFE shown by this material is also not observed in the category 3 particles (the absorption band at $1,252 \mathrm{~cm}^{-1}$ shown by RB-QD040047-02 cannot be related to PTFE because of the absence of fluorine indicated by F-XANES).

\section{Comparison between category 3 particles and artificial substances}

Raman $G$-band diagram and thermal maturity

Figure 9 shows a diagram of G-band parameters. Thermal metamorphism causes ordering of the IOM and shifts its data point toward the lower right in this diagram (e.g., Sandford et al. 2006; Busemann et al. 2007). Although low-temperature carbon thermometers based on Raman spectroscopy should be restricted to a given geological context (e.g., Cody et al. 2008; Quirico et al. 2009), Raman data are fingerprints of precursors as well as certain metamorphic conditions (Quirico et al. 2009). The G-band positions of the three category 3 particles

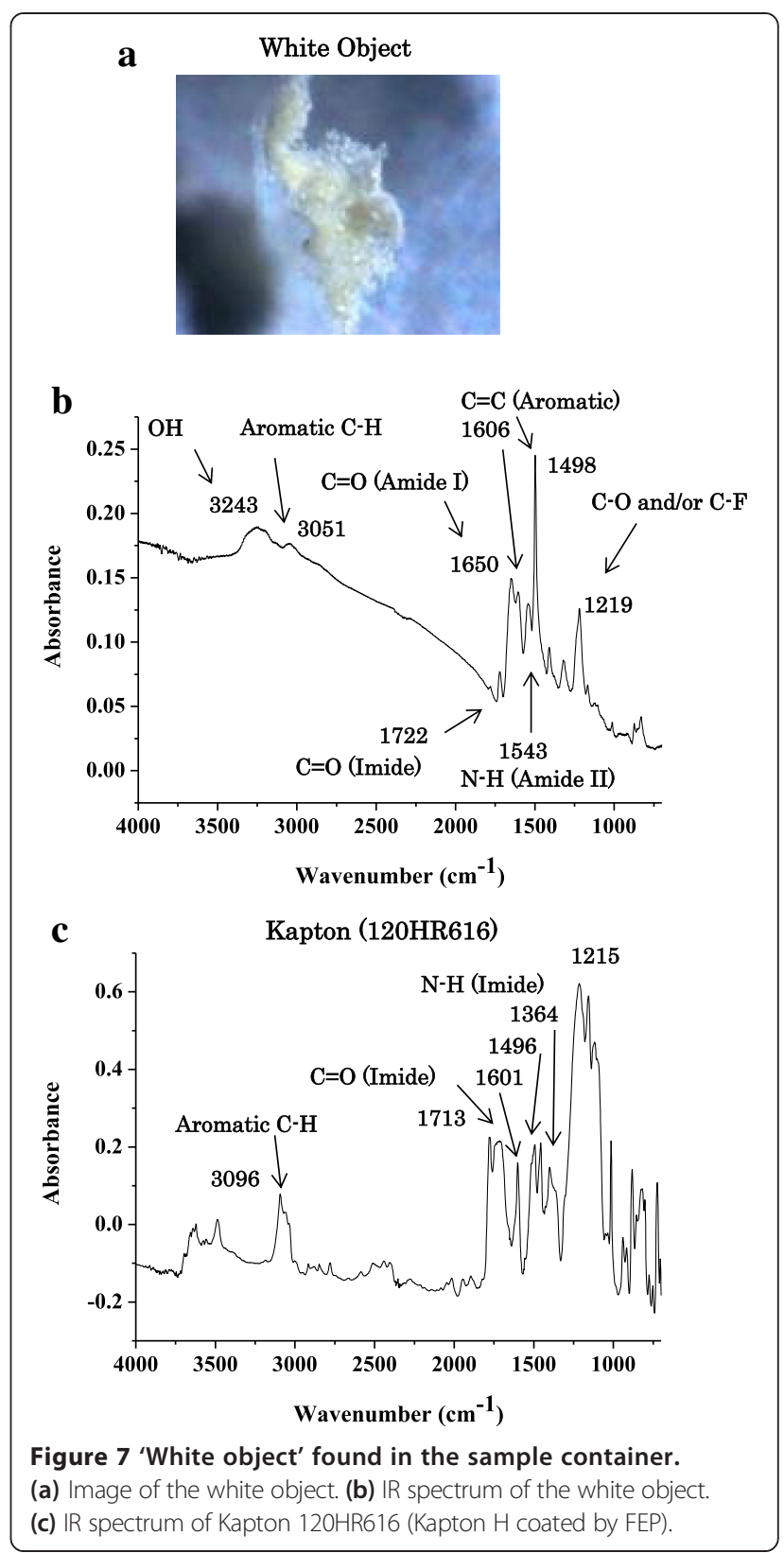

are located in a more disordered region than that of the chondritic primitive IOM. The FWHMs-G (full width in the half maximum) are broad (larger than $80 \mathrm{~cm}^{-1}$ ). It seems unsuitable to the LL4-6 parent body because FWHMs-G of even the IOMs in CO3.7 and CV3.7 have smaller band widths than $60 \mathrm{~cm}^{-1}$ (Busemann et al. 2007). The category 3 particles seem to be grouped together with the artifact produced from the Viton glove after laser exposure, rather than being associated with the chondritic IOM. The particles seem to be at a low maturity level, suggesting that they have not experienced strong heating, provided that they have not suffered serious irradiation-induced increase of disorder by NanoSIMS 


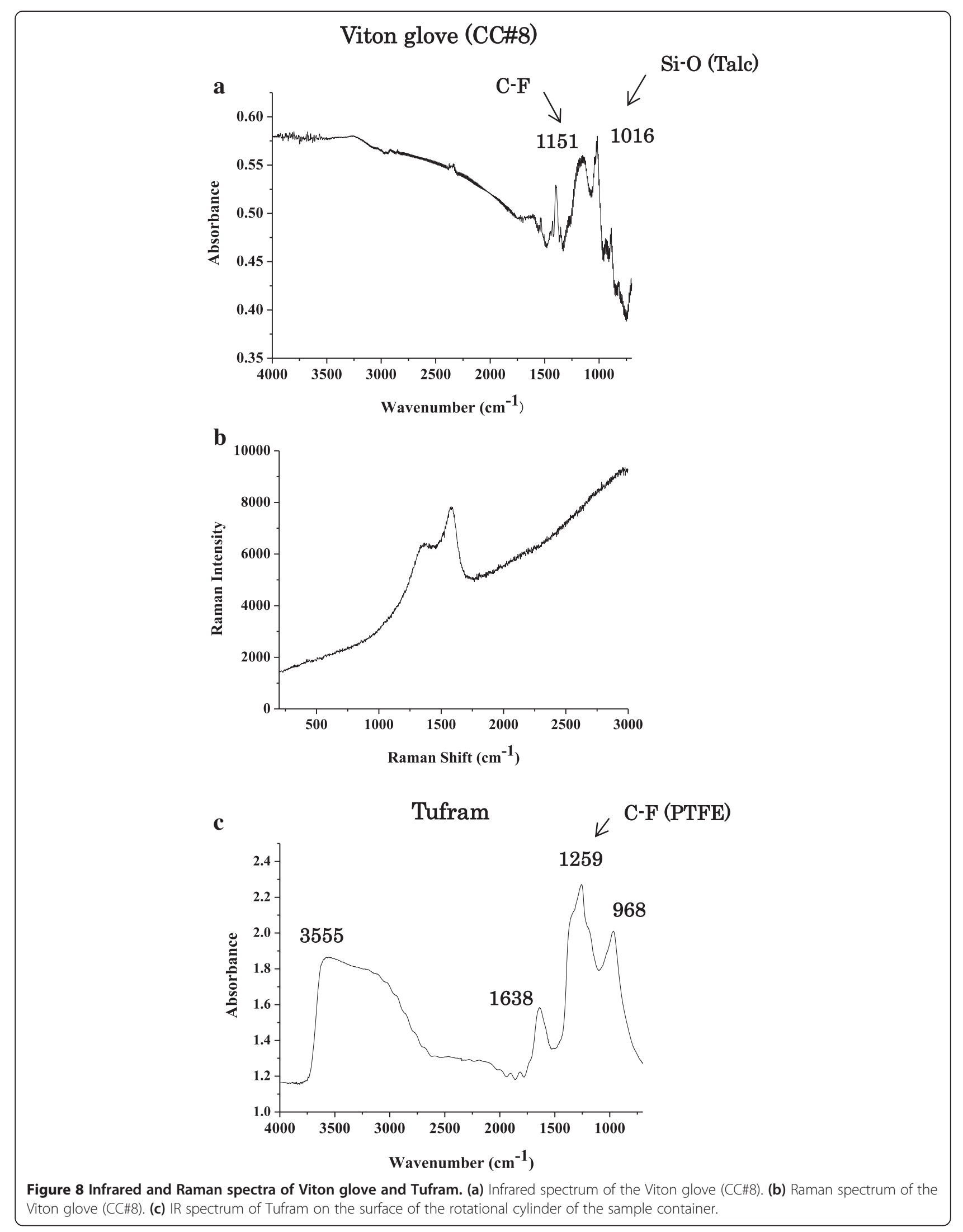




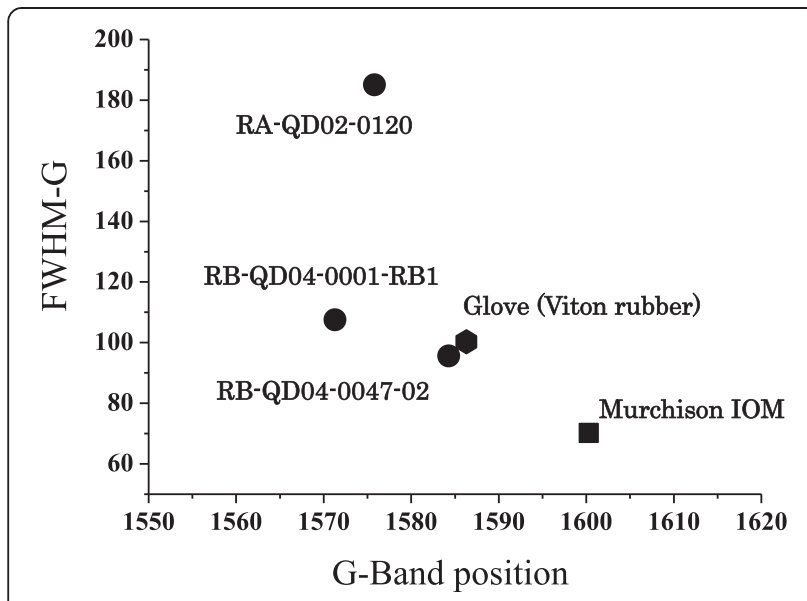

Figure 9 Raman G-band diagram. Spectra of the position (a) of the particle RB-QD04-0047-02 (Figure 2) and the position (a) of RB-QD040001_RB01 (Figure 3) were used for the calculations of G-band parameters.

analysis prior to this investigation. C-XANES spectra also show the lack of the $1 \mathrm{~s}-\sigma^{*}$ exitons at $291.6 \mathrm{eV}$ that is generally observed from thermally metamorphosed type $3+$ chondrites (Yabuta et al. 2014), suggesting that an origin for the particles from the LL4-6 parent body is unlikely.

\section{The category 3 particles as thermally unaltered materials}

$\mathrm{H}, \mathrm{C}$, and $\mathrm{N}$ isotopic compositions can be sensitive indicators for determining the provenance of primitive extraterrestrial carbonaceous matter. The three category 3 particles however have been reported to show terrestrial $\mathrm{H}, \mathrm{C}$, and $\mathrm{N}$ isotopic compositions within errors, and micrometer-sized hot spots with anomalous $\mathrm{H}, \mathrm{C}$, and $\mathrm{N}$ compositions have not been found (Ito et al. 2014). However, these observations are not enough to determine whether the particles are terrestrial contaminants or extraterrestrial matter, because isotopically normal carbonaceous material has been also reported in IDPs and Antarctic micrometeorites (Messenger 2000; Yabuta et al. 2013). When isotopic anomalies are not found, a simple and conclusive method to determine whether a carbonaceous substance is extraterrestrial has not yet been established, excluding the materials that would show enantiomer excess such as amino acids and monosaccharides. If it cannot be identified as a manmade substance, it should be confirmed that the elemental composition, structure, and provenance are consistent with extraterrestrial origin.

The IR spectra of the two category 3 particles, RBQD04-0047-02 and RB-QD04-0001, seem not to have the characteristics of the unheated chondritic IOM. Further, the spectral features of the two particles are not similar to known extraterrestrial carbonaceous matter heated or unheated. In addition, no IR spectra of artificial carbonaceous substances including the white object show exactly the same spectral features as the category 3 particles. Although it is difficult to determine definitively whether the particles are extraterrestrial in origin based on these limited results, their terrestrial origin(s) is/are suggested. They may be some reaction products from such artificial substances; however, the lack of C-F stretching indicates that all the category 3 particles are not 'fresh' contaminants from Viton, PTFE, and Tufram derived from the curation facilities.

However, the IR spectrum of RB-QD04-0047-02 has several absorption bands in common with those of the white object (Figure 10). The carbonyl absorption at $1,729 \mathrm{~cm}^{-1}$ suggests $\mathrm{C}=\mathrm{O}$ stretching of imide, $1,685 \mathrm{~cm}^{-1}$ absorption suggests $\mathrm{C}=\mathrm{O}$ stretching of amide (amide $\mathrm{I}$ ), $1,506 \mathrm{~cm}^{-1}$ suggests aromatic $\mathrm{C}=\mathrm{C}$ stretching, and $1,252 \mathrm{~cm}^{-1}$ may be $\mathrm{C}-\mathrm{O}$ stretching. This concordance suggests that RB-QD04-0047-02 has the same structural units as those of the polyimide/polyamide resin, suggesting it is a reaction product of polyimide resin. RB-QD04-0047-02 is enriched in imide structure more than the white object. Therefore, RB-QD04-0047-02 is a material that has a common starting material to the white object, rather than a direct product from the white object. The decrease of aromatic $\mathrm{C}=\mathrm{C}$ at $1,498 \mathrm{~cm}^{-1}$ and extinction of the $1,219 \mathrm{~cm}^{-1}$ absorption may suggest elimination of the arylether and/or hydrazide unit(s). Alternatively, the extinction of the $1,219 \mathrm{~cm}^{-1}$ absorption band may be derived from the release of the coating resulting in the decrease of the $\mathrm{C}-\mathrm{F}$ absorption band, because some kinds of Kapton is coated with FEP. N-XANES analysis suggests the presence of nitrile and/or N-heterocycles (Yabuta et al. 2014). RB-QD04-0047-02 may contain such unit structures depicted in Figure 1 (compounds IV to VIII) and relates to polyimide/polyamide resin. RB-QD04-0001 shows aromatic $\left(1,509 \mathrm{~cm}^{-1}\right)$ and carbonyl $\left(1,684 \mathrm{~cm}^{-1}\right)$

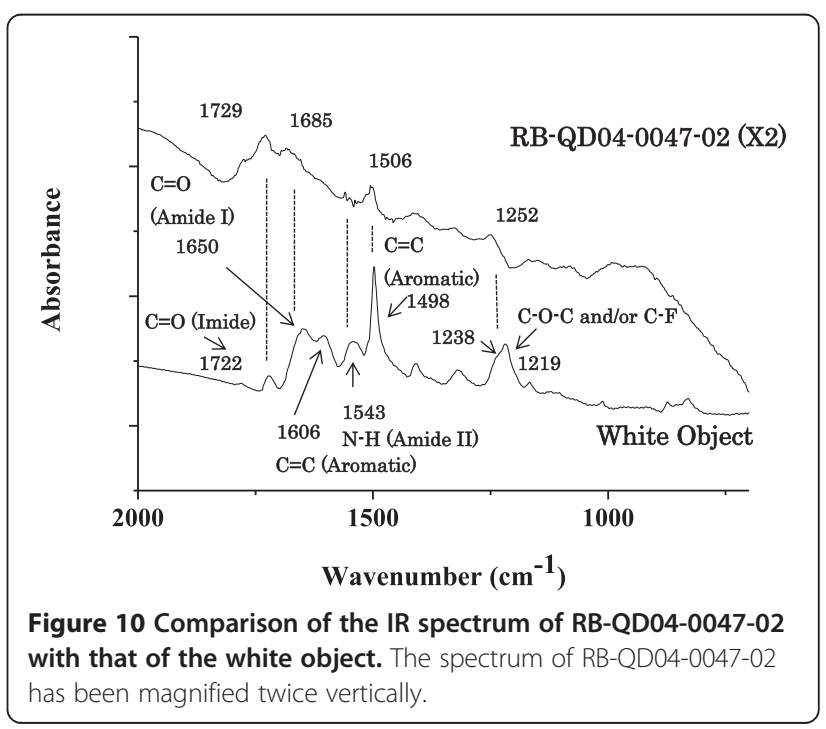


absorption bands. The carbonyl band can be assigned to amide as the $1,685 \mathrm{~cm}^{-1}$ absorption band of RB-QD040047-02, and it also may relate to the polyamide resin. Polyimide/polyamide resin originally does not have condensed graphene structure. The observed Raman bands are suggested to be the reaction products during sample return and/or artifacts that may be induced by electron beam exposure. In addition, the presence of $\mathrm{Si}$ in RBQD04-0047-02 (Uesugi et al. 2014; Naraoka et al. 2015) should be explained. It may be an addition like talc associated with Viton rubber.

RA-QD02-0120 does not seem to originate from a thermally altered parent body; however, IR and Raman data are not clear enough to clarify further its origin. The aliphatic C-H stretching of RA-QD02-0120, taking into account the presence of calcium carbonate (Uesugi et al. 2014; Yabuta et al. 2014), suggests various origins of the category 3 particles, although this particle belongs to the same subtype (blocky) as RB-QD04-0047-02. However, polyimide/polyamide resin is a major candidate for the source material of the particles. If several category 3 particles could be consumed, chemical degradation and analysis of the products using HPLC/ HRMS could be of great help. The behavior of the resin during sample return should be clarified in order to determine the origin(s) of the category 3 particles.

\section{Conclusion}

The Raman spectra of the category 3 particles showed both G- and D-bands. In the G-band diagram, the parameters plot in the disordered region, suggesting that the three category 3 particles, RB-QD04-0047-02, RBQD04-0001, and RA-QD02-0120, are not thermally altered substances. The particles are not likely to have originated from an LL4-6 parent asteroid. The particles plot close to the laser-induced artifact of Viton rubber rather than the Murchison IOM. The Raman results of the particles are not similar to known extraterrestrial carbonaceous matter heated or unheated. The IR spectral features of the RB-QD04-0047-02 and RB-QD040001 are also not similar to the Murchison IOM. The IR result of RA-QD02-0120 is not sufficiently clear. Although it cannot be determined whether they are extraterrestrial or not by these limited results, their terrestrial origin is suggested.

They were not identified as being the same as the artificial contaminants examined in this investigation. The lack of C-F stretching indicates the category 3 particles are not 'fresh' contaminants of Viton, PTFE, and Tufram at the curation facilities. They may be reaction products from artificial substances exposed to radiation during the sample return. In particular, RB-QD04-0047-2 may be a mixture of polyimide/polyamide resin as is the white object. It has several IR spectral absorption bands in common with the white object. It may relate to a degradation of polyimide/polyamide resin. RB-QD04-0001 may also relate to the resin. Although RA-QD02-0120 suggests a different origin and the category 3 particles may have various origins, it is necessary to clarify the behavior of polyimide/polyamide resin during sample return in order to determine the origin(s) of the category 3 particles.

\section{Competing interests}

The authors declare that they have no competing interests.

\section{Authors' contributions}

FK contributed to the IR and Raman analyses and image data of the samples except RA-QD02-0120, interpretation, and manuscript preparation. MU performed the IR and Raman analyses of RA-QD02-0120. MU, YK, YI, TY, $M A$, and AF contributed to the sample preparation. HN, MI, HY, HM, YT, and TO participated in the design of the research and interpretation. All authors read and approved the final manuscript.

\section{Acknowledgements}

We appreciate the two anonymous reviewers for their comments to improve the manuscript. We also thank to official editor Trever Ireland for helpful comments and editorial handling.

\section{Author details}

${ }^{1}$ Department of Earth and Planetary Sciences, Faculty of Science, Kyushu University, Hakozaki, Fukuoka 812-8581, Japan. ${ }^{2}$ Institute of Space and Astronautical Science, Japan Aerospace Exploration Agency, 3-1-1, Yoshinodai, Sagamihara, Kanagawa 252-5210, Japan. ${ }^{3}$ Kochi Institute for Core Sample Research, Japan Agency for Marine-Earth Science Technology, 200, Monobe Otsu, Nankoku, Kochi 783-8502, Japan. ${ }^{4}$ Department of Earth and Space Science, Osaka University, 1-1, Machikaneyama, Toyonaka, Osaka 560-0043, Japan. ${ }^{5}$ Life, Environment and Materials Science, Fukuoka Institute of Technology, 3-30-1, Wajiro-higashi, Fukuoka 811-0295, Japan. ${ }^{6}$ Institute of Biogeosciences, Japan Agency for Marine-Earth Science and Technology, 2-15, Natsushima-Cho, Yokosuka 237-0061, Japan.

Received: 1 May 2014 Accepted: 6 January 2015

Published online: 11 February 2015

\section{References}

Abe M, Takagi Y, Kitazato K, Abe S, Hiroi T, Vilas F, Clark BE, Abell PA, Lederer SM, Jarvis KS, Nimura T, Ueda Y, Fujiwara A (2006) Near-infrared spectral results of asteroid Itokawa from the Hayabusa spacecraft. Science 312:1134-1338

Busemann H, Alexander CMO'D, Nittler LR (2007) Characterization of insoluble organic matter in primitive meteorites by microRaman spectroscopy. Meteorit Planet Sci 42:1387-1416

Cody GD, Alexander CMO'D, Yabuta H, Kilcoyne ALD, Araki T, Ade H, Dera P, Fogel M, Militzer B, Mysen BO (2008) Organic thermometry for chondritic parent bodies. Earth Plant Sci Lett 272:446-455

Ebihara M, Sekimoto S, Shirai N, Hamajima Y, Yamamoto M, Kumagai K, Oura Y, Ireland TR, Kitajima F, Nagao K, Nakamura T, Naraoka H, Noguchi T, Okazaki R, Tsuchiyama A, Uesugi M, Yurimoto H, Zolensky ME, Abe M, Fujimura A, Mukai T, Yada T (2011) Neutron activation analysis of a particle returned from asteroid Itokawa. Science 333:1119-1121

Hiroi T, Abe M, Kitazato K, Abe S, Clark BE, Sasaki S, Ishiguro M, Barnouin-Jha OS (2006) Developing space weathering on the asteroid 25143 Itokawa. Nature 443:56-58

Ito M, Uesugi M, Naraoka H, Yabuta H, Kitajima F, Mita H, Takano Y, Karouji Y, Yada T, Ishibashi Y, Okada T, Abe M (2014) H, C and N isotopic compositions of Hayabusa category 3 organic samples. Earth, Planet Space 66:102

Kebukawa Y, Nakashima S, Zolensky ME (2010) Kinetics of organic matter degradation in the Murchison meteorite for the evaluation of parent-body temperature history. Meteorit Planet Sci 45:99-113

Kebukawa Y, Alexander CMO'D, Cody GD (2011) Compositional diversity in insoluble organic matter in type 1,2 and 3 chondrites as detected by infrared spectroscopy. Geochim Cosmochim Acta 75:3530-3541 
Kitajima F, Kotsugi M, Ohkochi T, Naraoka H, Ishibashi Y, Abe M, Fujimura A, Okazaki R, Yada T, Nakamura T, Noguchi T, Nagao K, Tsuchiyama A, Yurimoto H, Ebihara M, Mukai T, Sandford SA, Okada T, Shirai K, Ueno M, Yoshikawa M, Kawaguchi J (2011) A preliminary micro-spectroscopic analysis of the carbonaceous matter in the particles recovered by the Hayabusa mission. 74th Annual Meeting of the Meteoritical Society, abstract \#5341, London, 8-12 August 2011

Makjanic J, Vis RD, Hovenier JW, Heymann D (1993) Carbon in the matrices of ordinary chondrites. Meteoritics 28:63-70

Messenger S (2000) Identification of molecular-cloud material in interplanetary dust particles. Nature 404:968-971

Nagao K, Okazaki R, Nakamura T, Miura YN, Osawa T, Bajo K, Matsuda S, Ebihara M, Ireland TR, Kitajima F, Naraoka H, Noguchi T, Tsuchiyama A, Yurimoto H, Zolensky ME, Uesugi M, Shirai K, Abe M, Yada T, Ishibashi Y, Fujimura A, Mukai T, Ueno M, Okada T, Yoshikawa M, Kawaguchi J (2011) Irradiation history of Itokawa regolith material deduced from noble gases in the Hayabusa samples. Science 333:1128-1131

Nakamura T, Noguchi T, Tanaka M, Zolensky ME, Kimura M, Tsuchiyama A, Nakato A, Ogami T, Ishida H, Uesugi M, Yada T, Shirai K, Fujimura A, Okazaki R, Sandford SA, Ishibashi Y, Abe M, Okada T, Ueno M, Mukai T, Yoshikawa M, Kawaguchi J (2011) Itokawa dust particles: a direct link between S-type asteroids and ordinary chondrites. Science 333:1113-1116

Naraoka H, Mita H, Hamase K, Mita M, Yabuta H, Saito K, Fukushima K, Kitajima F, Sandford SA, Nakamura T, Noguchi T, Okazaki R, Nagao K, Ebihara M, Yurimoto H, Tsuchiyama A, Abe M, Shirai K, Ueno M, Yada T, Ishibashi Y, Okada T, Fujimura A, Mukai T, Yoshikawa M, Kawaguchi J (2012) Preliminary organic compound analysis of microparticles returned from Asteroid 25143 Itokawa by the Hayabusa mission. Geochem J 46:61-72

Naraoka H, Aoki D, Fukushima K, Uesugi M, Ito M, Kitajima F, Mita H, Yabuta H, Takano Y, Yada T, Ishibashi Y, Karouji Y, Okada T, Abe M (2015) ToF-SIMS analysis of carbonaceous particles in the sample catcher of the Hayabusa spacecraft. Earth, Planet. Space. in press

Noguchi T, Nakamura T, Kimura M, Zolensky ME, Tanaka M, Hashimoto T, Konno M, Nakato A, Ogami T, Fujimura A, Abe M, Yada T, Mukai T, Ueno M, Okada T, Shirai K, Ishibashi Y, Okazaki R (2011) Incipient space weathering observed on the surface of Itokawa dust particles. Science 333:1121-1125

Oba Y, Naraoka H (2009) Elemental and isotope behavior of macromolecular organic matter from CM chondrites during hydrous pyrolysis. Meteorit Planet Sci 41:1175-1181

Quirico E, Montagnac G, Rouzaud J-N, Bonal L, Bourot-Denise M, Duber S, Reynard B (2009) Precursor and metamorphic condition effects on Raman spectra of poorly ordered carbonaceous matter in chondrites and coals. Earth Planet Sci Lett 287:185-193

Sandford SA, Aléon J, Alexander CMO'D, Araki T, Bajt S, Baratta GA, Borg J, Bradley JP, Brownlee DE, Brucato JR, Burcell MJ, Busemann $H$, Butterworth A, Clemett SJ, Cody G, Colangeli L, Cooper G, D'Hendecourt L, Djouadi Z, Dworkin JP, Ferrini G, Fleckenstein H, Flynn GJ, Fachi IA, Fries M, Gilles MK, Glavin DP, Gunelle M, Grossemy F, Jacobsen C, Keller LP, Kilcoyne ALD, Leitner J, Matrajt G, Meibom A, Mennella V, Mostefaoui S, Nittler LR, Palumbo ME, Papanastassiou DA, Robert F, Rotundi A, Snead CJ, Spencer MK, Stadermann FJ, Steele A, Stephan T, Tsou P, Tyliszczak T, Westphal AJ, Wirick S, Wopenka B, Yabuta H, Zare RN, Zolensky ME (2006) Organics captured from comet $81 \mathrm{p} /$ Wild 2 by the Stardust Spacecraft. Science 314:1720-1724

Tsuchiyama A, Uesugi M, Matsushima T, Michikami T, Kadono T, Nakamura T, Uesugi K, Nakano T, Sandford SA, Noguchi R, Matsumoto T, Matsuno J, Nagano T, Imai Y, Takeuchi A, Suzuki Y, Ogami T, Katagiri J, Ebihara M, Ireland TR, Kitajima F, Nagao K, Naraoka H, Noguchi T, Okazaki R, Yurimoto H, Zolensky ME, Mukai T, Abe M, Yada T, Fujimura A, Yoshikawa M, Kawaguchi J (2011) Three-dimensional structure of Hayabusa samples: origin and evolution of Itokawa regolith. Science 333:1121-1125

Ueno K, Hirai O, Takeuchi K, Masuda K, Ejiri T (2009) Characterization of polymers using selective degradation reactions (in Japanese). Hitachi Chemical Technical Report 53:23-26

Uesugi M, Naraoka H, Ito M, Yabuta H, Kitajima F, Takano Y, Mita H, Ohnishi I, Kebukawa Y, Yada T, Karouji Y, Ishibashi Y, Okada T, Abe M (2014) Sequential analysis of carbonaceous materials in Hayabusa-returned sample for the determination of their origin. Earth, Planet Space 66:102

Yabuta H, Noguchi T, Itoh S, Sakamoto N, Hashiguchi M, Abe K, Tsujimoto S, Kilcoyne ALD, Okubo A, Okazaki R, Tachibana S, Nakamura T, Terada K, Ebihara M, Nagahara H (2013) Evidence of minimum aqueous alteration in rock-ice body: update of organic chemistry and mineralogy of ultracarbonaceous Antarctic micrometeorite, 44th Lunar and Planetary Science Conference, Abstract \#2335, The Woodlands, Texas, 18-22 March 2013 Yabuta H, Uesugi M, Naraoka H, Ito M, Kilcoyne ALD, Sandford SA, Kitajima F, Mita H, Takano Y, Yada T, Karouji Y, Ishibashi Y, Okada T, Abe M (2014) X-ray absorption near edge structure spectroscopic study of Hayabusa category 3 carbonaceous particles. Earth, Planet Space 66:156

Yada T, Fujimura A, Abe M, Nakamura T, Noguchi T, Okazaki R, Nagao K, Ishibashi Y, Shirai K, Zolensky ME, Sandford S, Okada T, Uesugi M, Karouji Y, Ogawa M, Yakame S, Ueno M, Mukai T, Yoshikawa M, Kawaguchi J (2014) Hayabusa return sample curation in the Planetary Material Sample Curation Facility of JAXA. Meteorit Planet Sci 49:135-153

Yurimoto H, Abe K, Abe M, Ebihara M, Fujimura A, Hashiguchi M, Hashizume K, Ireland TR, Itoh S, Katayama J, Kato C, Kawaguchi J, Kawasaki N, Kitajima F, Kobayashi S, Meike T, Mukai T, Nagao K, Nakamura T, Naraoka H, Noguchi T, Okazaki R, Park C, Sakamoto N, Seto Y, Takei M, Tsuchiyama A, Uesugi M, Wakaki S, Yada T, Yamamoto K, Yoshikawa M, Zolensky ME (2011) Oxygen isotopic compositions of asteroidal materials returned from Itokawa by the Hayabusa mission. Science 333:1116-1119

\section{Submit your manuscript to a SpringerOpen ${ }^{\circ}$ journal and benefit from:}

- Convenient online submission

- Rigorous peer review

- Immediate publication on acceptance

- Open access: articles freely available online

- High visibility within the field

- Retaining the copyright to your article

Submit your next manuscript at $>$ springeropen.com 